REVISTA

\title{
MÉTODO NÃO DESTRUTIVO PARA DETERMINAÇÃO DA ÁREA FOLIAR DE Turnera subulata
}

\section{NON-DESTRUCTIVE METHOD FOR DETERMINING THE LEAF AREA OF Turnera subulata}

\author{
Aline Queiroz Castro ${ }^{1}$ \\ Gabriel dos Santos Conceição ${ }^{2}$ \\ Gabriela Singulani de Brito ${ }^{3}$ \\ Quezia Barbosa dos Santos ${ }^{4}$ \\ Jasmyn Tognere ${ }^{5}$ \\ Vinicius de Souza Oliveira ${ }^{6 *}$ \\ Sara Dousseau Arantes ${ }^{7}$ \\ Omar Schmildt ${ }^{8}$ \\ Edilson Romais Schmildt ${ }^{9}$ \\ *Autor para correspondência

\begin{abstract}
${ }^{1}$ Universidade Federal do Espírito Santo, CEUNES, ES, Brasil; alinecastros2@hotmail.com ${ }^{2}$ Universidade Federal do Espírito Santo, CEUNES, ES, Brasil; gabrieldossantosconceicao@gmail.com ${ }^{3}$ Universidade Federal do Espírito Santo, CEUNES, ES, Brasil; gabisingulani15@gmail.com ${ }^{4}$ Universidade Federal do Espírito Santo, CEUNES, ES, Brasil; queziabarbosa74@gmail.com

${ }^{5}$ Universidade Federal do Espírito Santo, CEUNES, ES, Brasil; tognerejasmyn@gmail.com

${ }^{6}$ Universidade Federal do Espírito Santo, CCAE, ES, Brasil; souzaoliveiravini@gmail.com ${ }^{7}$ Instituto Capixaba de Pesquisa, Assistência Técnica e Extensão Rural (INCAPER), Linhares, ES, Brasil. Email: saradousseau@gmail.com

${ }^{8}$ Universidade Federal do Espírito Santo, CEUNES, ES, Brasil; omarschmildt@gmail.com ${ }^{9}$ Universidade Federal do Espírito Santo, CEUNES, ES, Brasil; e.romais.s@gmail.com

*Autor de correspondência
\end{abstract}

Artigo submetido em 15/03/2021, aceito em 18/04/2021 e publicado em 24/05/2021.

Resumo: Turnera subulata é uma planta medicinal que tem demostrado associação com o processo de cicatrização. A determinação da área foliar é importante parâmetro em estudos agronômicos, fisiológicos e morfológicos das diversas espécies vegetais. Essa determinação pode ser feita de forma destrutiva ou não destrutiva. Dentre os métodos não destrutivos se destaca a uso de equações matemáticas que relacionam a área foliar e as medidas lineares das folhas como o comprimento, largura e sua combinação. Desta forma, objetivou-se com este estudo ajustar e validar uma equação matemática que estima a área foliar de T. subulata de forma não destrutiva. Assim, com base em 350 folhas, foram ajustadas equações de modelo lineares de primeiro grau, quadrático e potência, em que a área foliar observada foi utilizada como variável dependente em função das medidas lineares do comprimento, largura e produto da multiplicação do comprimento com a largura. Todas as equações ajustadas foram validadas através de critérios apropriados a partir de uma amostra de 100 folhas separadas unicamente para esta finalidade. Desse modo, a equação de modelo quadrático 
$\mathrm{AFE}=0.1578612+0,6754851(\mathrm{CL})-0.0005830(\mathrm{CL})^{2}$ obtida a partir do produto da multiplicação do comprimento com a largura é a mais adequada para estimar a área foliar de $T$. subulata de maneira simples e precisa.

Palavras-chave: equações matemáticas; modelagem estatística; medidas lineares.

\begin{abstract}
Turnera subulata is a medicinal plant that has been shown to be associated with the healing process. The determination of leaf area is an important parameter in agronomic, physiological and morphological studies of different plant species. This determination can be made in a destructive or non-destructive way. Among the non-destructive methods, the use of mathematical equations that relate the leaf area and the linear measurements of the leaves, such as length, width and their combination, stands out. Thus, the objective of this study was to adjust and validate a mathematical equation that estimates the leaf area of $T$. subulata in a non-destructive way. based on 350 leaves, linear first-degree, quadratic and power model equations were adjusted, in which the observed leaf area was used as a dependent variable depending on the linear measures of length (L), width (W) and product of the multiplication of length with width (LW). All adjusted equations were validated using appropriate criteria from a sample of 100 sheets separated solely for this purpose. In this way, the quadratic model equation $E L A=0.1578612+0.6754851$ ( $(\mathrm{W})-0.0005830$ ( $\mathrm{LW})^{2}$ obtained from the product of the multiplication of the length with the width is the most adequate to estimate the leaf area of T. subulata in a simple and precise way.
\end{abstract}

Keywords: mathematical equations; statistical modeling; linear measurements.

\section{INTRODUÇÃO}

Turnera subulata, conhecida popularmente como Muchinga, Flor do Guarujá e Chanana, é uma espécie de herbácea perene nativa da Flórida, México, América Central e América do Sul, sua altura vária de 30 a $80 \mathrm{~cm}$, com folhas simples ovaladas, face superior marcada por nervações e margens serreadas, podendo ou não conter estípulas. Possui flores formadas por pétalas que variam do amarelo a brancas-amareladas de cor marrom na base que se abrem logo pela manhã, depois de receber a luz direta do sol, e se fecham após o meio-dia (GOUVEIA et al., 2013). Considerada uma planta medicinal, a $T$. subulata vem sendo utilizada em pesquisas sobre o processo de cicatrização em feridas cutâneas, onde vários extratos da planta têm se mostrado promissores no processo (FALEIRO et al., 2009).

Em relação a área foliar, sua determinação pode ser realizada por métodos diretos e indiretos (MABROUK; CARBONNEAU, 1996). Os métodos não destrutivos diretos caracterizam-se por serem rápidos, precisos e seus resultados serem expressos imediatamente com o uso de equipamentos portáteis (BINDI et al., 1997; DOBROWSKI et al., 2002), porém sua utilização é restrita devido ao alto custo de aquisição (OLIVEIRA et al., 2002) e há necessidade de manutenção e/ou ajustes frequentes nos equipamentos. Os métodos não destrutivos indiretos são caracterizados por relações entre fatores, como área foliar e medições lineares realizadas em folhas. Estas relações são expressas em geral por equações de regressão que são utilizadas na estimativa da área foliar (PEDRO JÚNIOR et al., 1986; RAMKHELAWAN; BRATHWAITE, 1990; OLIVEIRA et al., 2002).

O uso de equações matemáticas para estimar a área foliar já foi relatada na literatura para diversas espécies (POMPELLI et al., 2012; SCHMILDT et al., 2015; MONTOLATTO et al., 2020; OLIVEIRA et al., 2017; OLIVEIRA et al., 2019; OLIVEIRA et al., 2020; SANTOS et al., 2020; TEOBALDELLI et al., 2020), entretanto, não se encontrou estudos que envolvam esta técnica para estimar a área foliar de T. subulata. 
Desta forma, objetivou-se com este estudo ajustar, validar e indicar uma equação matemática que estime a área de $T$. subulata de forma não destrutiva através das dimensões foliares do comprimento e da largura.

\section{MATERIAIS E MÉTODOS}

O estudo foi realizado com folhas de Turnera subulata, coletadas no município de São Mateus, Norte do Estado do Espírito Santo, Brasil, localizado a $18^{\circ} 40^{\prime} 36^{\prime \prime}$ de latitude Sul e $39^{\circ} 51^{\prime} 35^{\prime}$ ' de longitude Leste. Foram cotadas 450 folhas de diferentes plantas em todos os estados de desenvolvimento que se mostravam ausentes de danos ou ataques de doenças ou pragas (OLIVEIRA et al., 2017).

Em laboratório, as folhas foram digitalizadas através de scanner de mesa modelo Photosmart $\mathrm{C} 4280^{\circledR}$, sendo as imagens salvas no formado TIFF (Tag Image File Format) com 75 dpi e processadas com o auxílio do software Image $^{\circledR}$ onde determinou-se 0 maior comprimento (C) ao longo da nervura principal, a largura máxima (L) do limbo foliar de cada folha, ambas as medidas em cm (Figura 1) e a área foliar observada (AFO) em $\mathrm{cm}^{2}$. Posteriormente, também foi determinado o produto da multiplicação do comprimento com a largura $(\mathrm{CxL}), \mathrm{em}^{\mathrm{cm}}{ }^{2}$.

Figura 1. Representação da medida do comprimento (C) ao longo da nervura principal e largura máxima (L) do limbo foliar de folhas de Turnera subulata

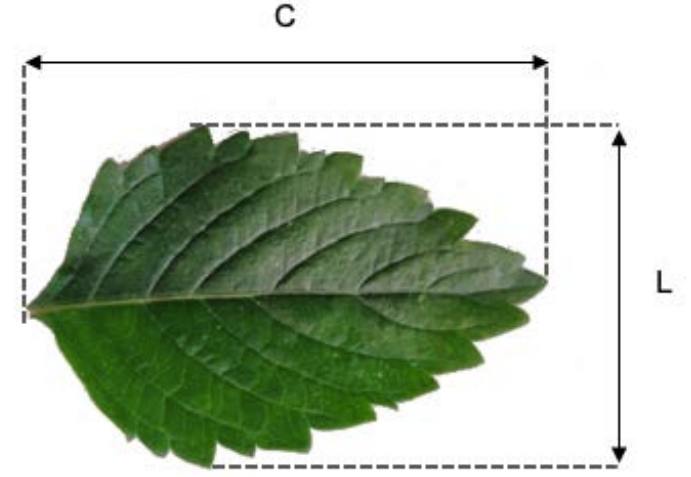

Fonte: Autores.

Para a modelagem, 350 folhas foram usadas, onde a AFO era a variável dependente (y) em função de $\mathrm{C}$, L e CxL como variáveis independentes (x). Os modelos lineares de primeiro grau representado por área foliar estimada $(\mathrm{AFE})=\widehat{\beta}_{0}+\widehat{\beta}_{1} \mathrm{x}$, quadrático representado por $\mathrm{AFE}=\widehat{\beta}_{0}+\hat{\beta}_{1} \mathrm{x}+\hat{\beta}_{2} \mathrm{x}^{2}$ e potência representado por $\mathrm{AFE}=\widehat{\beta}_{0} \mathrm{X}^{\widehat{\beta}_{1}}$, e seu respectivo coeficiente de determinação $\left(\mathrm{R}^{2}\right)$ foram obtidos.

Para a validação, 100 folhas separadas para esta finalidade tiveram os valores de C, L e CxL substituídos nas equações obtidas na modelagem, obtendo, assim, AFE, em $\mathrm{cm}^{2}$ por cada modelo. As médias de AFO e AFE foram comparadas pelo teste $t$ de Student a $5 \%$ de probabilidade. Também se determinou como critério de validação o erro absoluto médio (EAM), a raiz do quadrado médio do erro (RQME) e o índice d de Willmott (WILLMOTT, 1981), para todas as equações através das seguintes expressões:

$$
\begin{gathered}
E A M=\frac{\sum_{i=1}^{n}|A F E-A F O|}{n} \\
R Q M E=\sqrt{\frac{\sum_{i=1}^{n}(A F E-A F O)^{2}}{n}} \\
d=1-\left[\frac{\sum_{i=1}^{n}(A F E-A F O)^{2}}{\sum_{i=1}^{n}(|A F E-\overline{A F O}|+|A F O-\overline{A F O}|)^{2}}\right]
\end{gathered}
$$

Onde, AFE corresponde aos valores estimados da área foliar; AFO são os valores observados de área foliar; $\overline{\mathrm{AFO}}$ é a 
média dos valores da área foliar observada; e n é o número de folhas amostradas para a validação $(n=100)$.

Os critérios utilizados para a seleção do melhor modelo que estime a área foliar da $T$. subulata em função do C, L e CxL foram: a não significância entre 0 comparativo das médias de AFE e AFO; EAM e RQME mais próximo a zero e índice d mais próximos de um. As análises estatísticas foram realizadas com auxílio do software R (R CORE TEAM, 2020), através do pacote de dados ExpDes.pt versão 1.2 (FERREIRA et al., 2018).

\section{RESULTADOS E DISCUSSÃO}

Na Tabela 1 está representada a estatística descritiva para as características das folhas de $T$. subulata utilizadas para a modelagem e para a validação. Nota-se, que não há extrapolação dos valores amostrais utilizados para a validação em relação aos utilizados para a modelagem. Esse fato é importante, pois, folhas na validação com área abaixo ou acima das apresentadas no ajuste das equações podem gerar valores superestimados ou subestimados, essa mesma constatação deve ser levada em consideração em futura utilização destas equações (LEVINE et al., 2017). Foi observado altos valores de desvio padrão (DP) e coeficiente de variação (CV) tanto na amostra utilizada para a modelagem quanto na amostra utilizada para a validação. Esse resultado, segundo Montolatto et al. (2020), é explicado pela diversidade de tamanhos das folhas presentes na amostragem.

Tabela 1. Valor mínimo, máximo e médio, desvio padrão (DP) e coeficiente de variação (CV) das variáveis: comprimento (C); largura (L); produto do comprimento com a largura (CxL) e área foliar observada (AFO) de folhas de Turnera subulata utilizadas para a modelagem e para a validação

\begin{tabular}{ccccccc}
\hline Variável & Unidade & Mínimo & Máximo & Média & DP & CV \\
\hline \multicolumn{7}{c}{350 folhas usadas para a modelagem } \\
\hline C & $\mathrm{cm}$ & 2,12 & 10,98 & 6,21 & 1,63 & 26,36 \\
L & $\mathrm{cm}$ & 1,35 & 6,74 & 3,88 & 0,90 & 23,21 \\
AFO & $\mathrm{cm}^{2}$ & 3,18 & 66,96 & 25,36 & 11,39 & 44,94 \\
\hline & $\mathrm{cm}^{2}$ & 2,24 & 43,01 & 16,84 & 7,35 & 43,67 \\
\hline C & $\mathrm{cm}$ & 2,13 & 7,37 & 4,36 & 1,22 & 28,02 \\
L & $\mathrm{cm}$ & 1,47 & 4,54 & 2,86 & 0,73 & 25,68 \\
CxL & $\mathrm{cm}^{2}$ & 3,41 & 30,72 & 13,27 & 6,69 & 50,45 \\
AFO & $\mathrm{cm}^{2}$ & 2,44 & 21,09 & 9,02 & 4,45 & 49,36 \\
\hline
\end{tabular}

Fonte: Autores. 
Embora os resultados mostrem boa relação entre a $\mathrm{AFO}$ e $\mathrm{C}$, $\mathrm{L}$ e CxL para todos os modelos de equação ajustada com coeficiente de determinação $\left(\mathrm{R}^{2}\right)$ superior a 0,90 , as equações ajustadas com base em $\mathrm{CxL}$ se mostraram mais precisas com $\mathrm{R}^{2}$ acima de 0,99, demostrando que mais de 99\% desta variável pode explicar os valores da área das folhas de $T$. subulata (Tabela 2). De fato, corroborando com os resultados encontrados neste estudo, a combinação de medidas lineares da superfície foliar, se mostrou mais adequada para Jatropha curcas (POMPELLI et al., 2012), Coffea canephora (SCHMILDT et al., 2015), Litchi chinensis Sonn. (OLIVEIRA et al., 2017), Maytenus obtusifolia Mart. (OLIVEIRA et al., 2019), Allagoptera arenaria (OLIVEIRA et al., 2020) e Tabebuia impetiginosa (Mart.) (SANTOS et al., 2020), sendo essas equações capazes de fornecer com maior exatidão a área foliar destas espécies.

Tabela 2. Equação com ajuste linear de primeiro grau, quadrático e potência e seu respectivo coeficiente de determinação $\left(\mathrm{R}^{2}\right)$ utilizando a área foliar observada (AFO) como variável dependente, em função do comprimento (C), largura (L) e produto do comprimento com a largura (CxL), de folhas de Turnera subulata

\begin{tabular}{ccc}
\hline Modelo & Equação & $\mathrm{R}^{2}$ \\
\hline Linear & $\mathrm{AFE}=-9,68319+4,27429(\mathrm{C})$ & 0,9034 \\
Linear & $\mathrm{AFE}=-13,2478+7,7479(\mathrm{~L})$ & 0,9013 \\
Linear & $\mathrm{AFE}=0,54228+0,64257(\mathrm{CL})$ & 0,9918 \\
Quadrático & $\mathrm{AFE}=-0,66824+1,06737(\mathrm{C})+0,26437(\mathrm{C})^{2}$ & 0,9175 \\
Quadrático & $\mathrm{AFE}=-4,23602+2,79075(\mathrm{~L})+0,64432(\mathrm{~L})^{2}$ & 0,9125 \\
Quadrático & $\mathrm{AFE}=0,1578612+0,6754851(\mathrm{CL})-0,0005830(\mathrm{CL})^{2}$ & 0,9920 \\
Potência & $\mathrm{AFE}=0,7471(\mathrm{C})^{1,6846}$ & 0,9175 \\
Potência & $\mathrm{AFE}=1,3220(\mathrm{~L})^{1,8468}$ & 0,9093 \\
Potência & $\mathrm{AFE}=0,7517(\mathrm{CL})^{0,9628}$ & 0,9920 \\
\hline
\end{tabular}

Fonte: Autores

Na Tabela 3 são apresentados os critérios utilizados na validação das equações ajustadas. Para todas as equações, não foram constatadas diferença significativa entre AFO e AFE de acordo com o intervalo de confiança de $95 \%$ pelo teste $\mathrm{t}$ de Student, demostrando que a área das folhas estimadas pelos modelos é similar à área das folhas observadas. Contudo, deve-se atentar que o erro gerado pelas equações na determinação da área foliar de T. subulata foi menor na equação de modelo quadrático obtida através de CxL, com valores do erro absoluto médio (EAM) e raiz quadrada média do erro (RQME) mais próximos ao nulo. Da mesma forma, para esse modelo de equação o valor do índice d de Willmott teve maior proximidade a um, o que sugere maior concordância entre a estimativa gerada pela equação e os valores reais observados. 
Tabela 3. Área foliar observada (AFO) e área foliar estimada (AFE) de equações lineares de primeiro grau, quadrática e potência para as variáveis independente comprimento (C), largura (L) e produto do comprimento com a largura (CxL), além do valor p, erro absoluto do erro (EAM), raiz quadrada médio do erro (RQME) e índice d de Willmott de folhas de Turnera subulata utilizadas para validação

\begin{tabular}{cccccccc}
\hline Modelo & Variável & AFO & AFE & Valor p & EAM & RQME & d \\
\hline Linear & C & & 8,9657 & 0,9344 & 1,3337 & 1,5976 & 0,9722 \\
Linear & L & & 8,9383 & 0,9080 & 1,6883 & 2,0541 & 0,9581 \\
Linear & CxL & & 9,0676 & 0,9431 & 0,3417 & 0,4587 & 0,9972 \\
Quadrático & C & & 9,4124 & 0,5336 & 1,0708 & 1,3042 & 0,9773 \\
Quadrático & L & 9,0229 & 9,3834 & 0,5871 & 1,1374 & 1,4780 & 0,9741 \\
Quadrático & CxL & & 8,9914 & 0,9603 & 0,3179 & 0,4397 & 0,9976 \\
Potência & C & & 9,3354 & 0,6202 & 1,0451 & 1,2834 & 0,9784 \\
Potência & L & & 9,6977 & 0,2896 & 1,0960 & 1,4715 & 0,9723 \\
Potência & CxL & & 9,0185 & 0,9944 & 0,3221 & 0,4406 & 0,9975 \\
\hline
\end{tabular}

Fonte: Autores.

Diante dos dados apresentados, a equação de modelo quadrático $\mathrm{AFE}=$ $0,1578612+0,6754851(\mathrm{CxL})-0,0005830$ obtida através do produto da multiplicação do comprimento com a largura é a mais adequada. A consistência destes resultados pode ser observada com a representação gráfica (Figura 2A), cujo valores reais da área foliar de $T$. subulata apresentaram boa distribuição em relação à linha da equação ajustada, além de distribuição normal dos residuais (Figura 2B) com 99,7\% dos dados compreendidos entre o intervalo de \pm 3 desvios padrões (ALMEIDA et al., 2019). Deve-se ressaltar, que o presente estudo foi realizado com plantas em condição de campo e com folhas sadias, sem o ataque de pragas ou doenças. Assim, caso se faça necessário o uso desta equação em outras condições diferente das deste estudo, essa deve ser validada sob tais condições para melhores estimativas (TEOBALDELLI et al., 2020). 
Figura 2. A) Equação de modelo quadrático e coeficiente de determinação $\left(R^{2}\right)$ e $\left.B\right)$ distribuição residual de folha de Turnera subulata utilizando a área foliar observada (AFO) como variável dependente, em função do produto do comprimento com a largura (CxL).

A

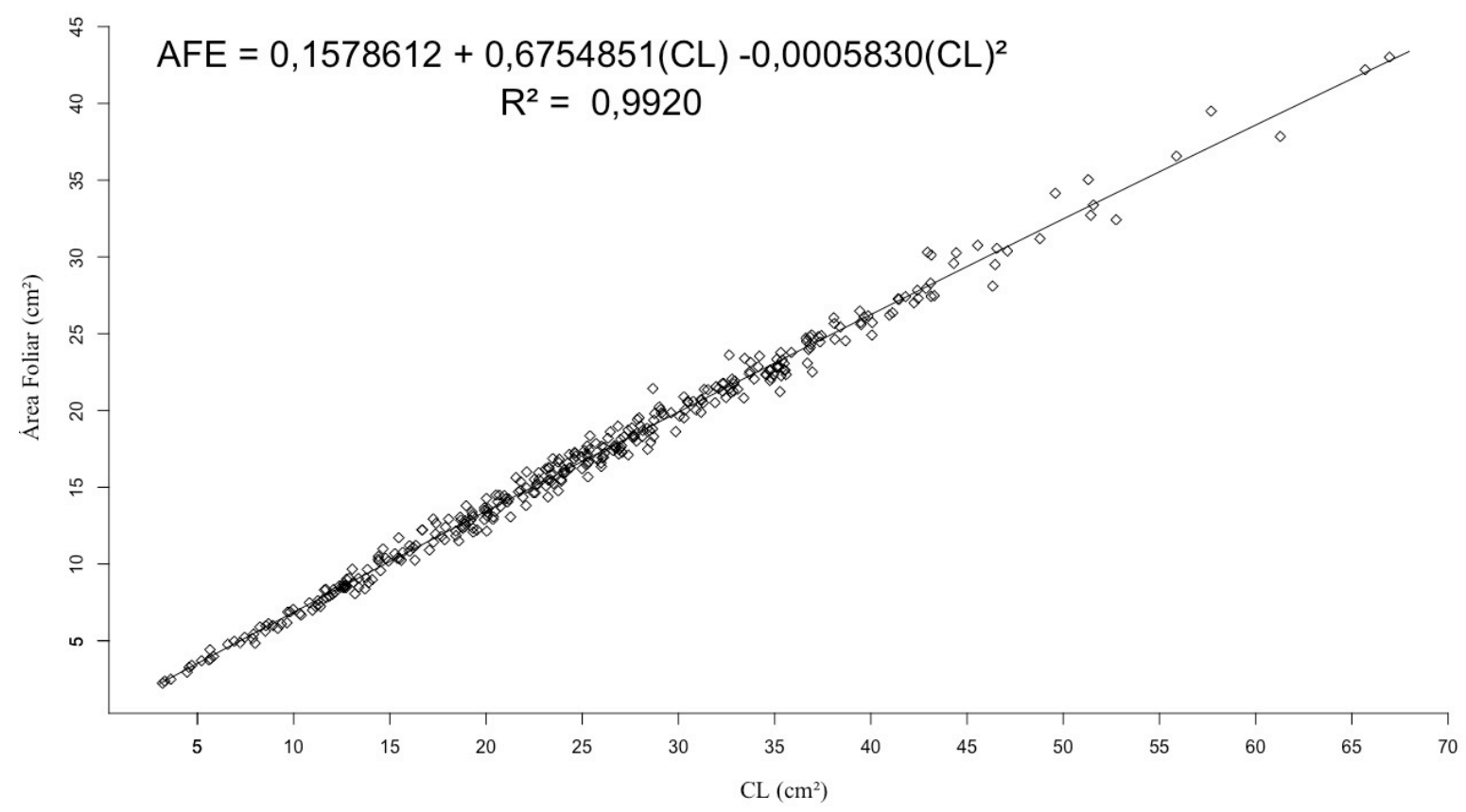

B

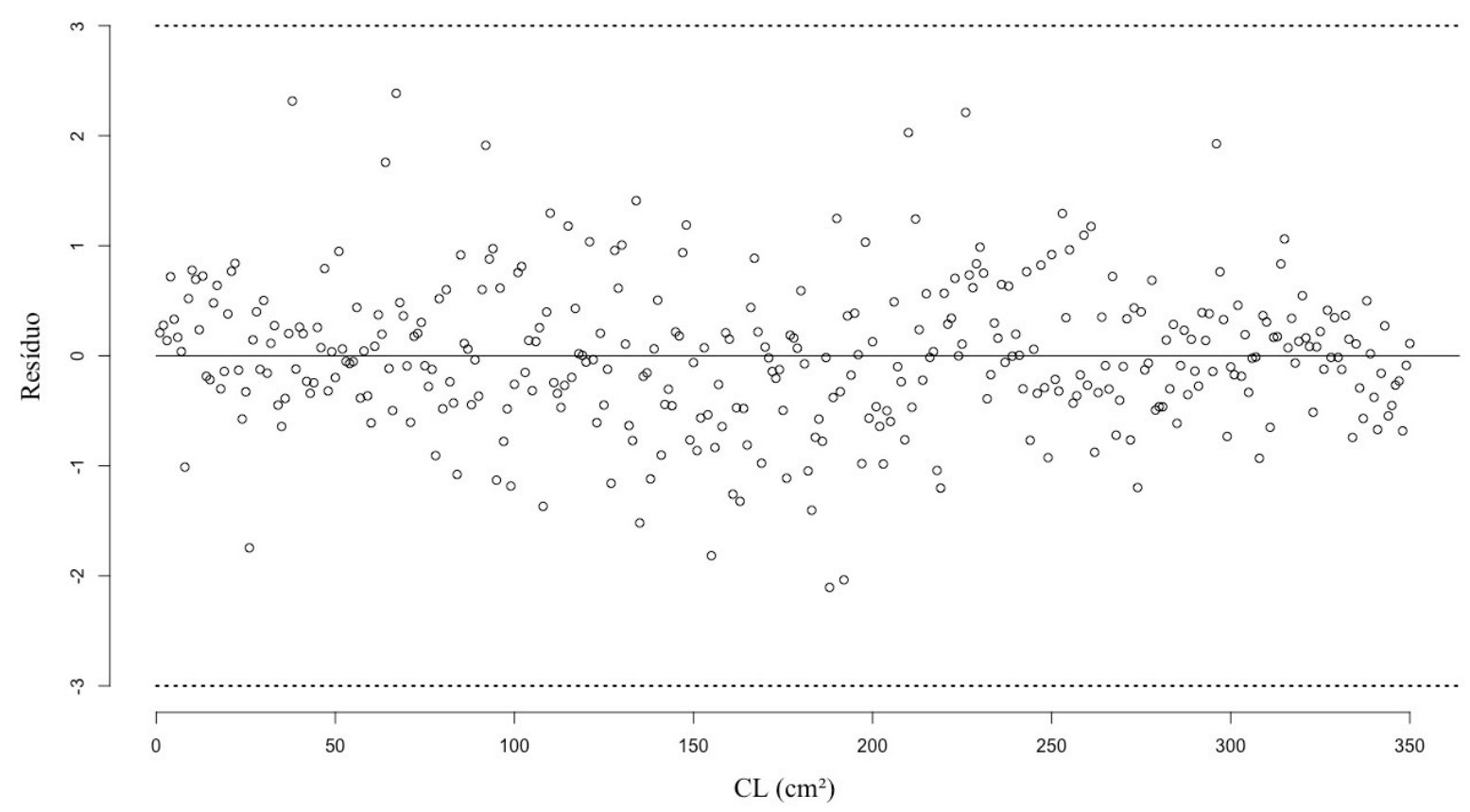

Fonte: Autores. 


\section{CONCLUSÃO}

A área foliar de Turnera subulata pode ser prevista de maneira simples e precisa através da equação de modelo quadrático AFE = 0,1578612 + 0,6754851 (CxL) 0,0005830 obtida através da multiplicação das medidas lineares das folhas comprimento e largura de maneira não destrutiva.

\section{REFERÊNCIAS}

ALMEIDA, C. O. Ensaios: Da amostra ao teorema do limite central: Um pouco dos fundamentos e uma aplicação prática. Cruz das Almas, BA: Embrapa Mandioca e Fruticultura, 40p., 2019.

BINDI, M.; MIGLIETTA, F.; GOZZINI, B.; ORLANDINI, S.; SEGHI, L. A simple model for simulation of growth and development in grapewine (Vitis vinifera). Model description. Vitis, Siebeldingen, v.36, n.2, p.67-71, 1997.

DOBROWSKI, S. Z.; USTIN, S. L.; WOLPERT, J. A. Remote estimation of vine canopy density invertically shootpositioned vineyards: determiningoptimal vegetation indexes. Australian Journal of Grape and Wine, v.8, n.2, p.117-125, 2002.

FALEIRO, C. C.; ELIAS, S. T. H.; CAVALCANTI, L. C.; CAVALCANTI, A. S. S. O extrato das folhas de babosa, Aloe vera na cicatrização de feridas experimentais em pele de ratos, num ensaio controlado por placebo. Natureza online, v.7, n.2, p.56-60, 2009.

FERREIRA, E. B.; CAVALCANTI, P. P. \& NOGUEIRA, D. A. Package 'ExpDes.pt'. 2018.

GOUVEIA, A. L. A.; SILVA, T. R. P. M.; SILVA, N. M. B.; MAGNATA, S. S. L. P. Análise da atividade cicatrizante da
Turnera subulata. Universidade Católica de Pernambuco, p. 1-9, 2013.

LEVINE, D. M.; STEPHAN, D. F.; SZABAT, K. A. Estatistic for managers using Microsoft Excel: global edition. (8th ed.) London: Person. 728p., 2017.

MABROUK, H.; CARBONNEAU, A. Une méthode simple de determination de la foliare de la vigne (Vitis vinifera L.). Progrès Agricole et Viticole, v.113, n.18, p.392-398, 1996.

MONTOLATTO, M. B.; VILLAMAGUAVERGANA, G. C.; CASTANHO, F. P.; KAWAKAMI, B.; ZERBINATO, B.; SILVA, M. A.; GUERRA, S. P. S. Models for leaf area estimation of three forest species in a short coppice rotation. Acta Ecologica Sinica, v. 40, n. 4, p. 263-267, 2020.

OLIVEIRA, M. N. S. de.; LOPES, P. S. N.; MERCADANTE, M. O.; OLIVEIRA, G. L.; GUSMÃOR, E. Medição da área foliar do pequizeiro utilizando asoma da nervura principal dos folíolos. Unimontes Científica, v.3, n.3, p. 1-7, 2002.

OLIVEIRA, P. S.; SILVA, W.; COSTA, A. A. M.; SCHMILDT, E. R.; VITÓRIA, E. L. Leaf area estimation in litchi by means of allometric relationships. Revista Brasileira de Fruticultura, 39(Special), p. 1-6, 2017.

OLIVEIRA, V. S.; SANTOS, K. T. H.; PINHEIRO, A. P. B.; SANTOS, G. P.; SANTOS, J. S. H.; CHISTÉ, H.; SCHMILDT, O.; ARANTES, S. D.; CZEPAK, M. P.; FERNANDES, A. A.; SCHMILDT, E. R. Modeling of the Leaf Area of Maytenus obtusifolia Mart. from Scanned Images. Agricultural Sciences, v. 10, n. 6, p. 796-806, 2019.

OLIVEIRA, V. S.; SILVA, J. V. G.; JARDIM A. S.; LONGUE, L. L.; AZEVEDO, A. L. R.; FERNANDES, A. A.; SANTOS, G. P.; SCHMILDT, O.; 
POSSE, R. P.; ARANTES, S. D.; SCHMILDT, E. R. Ajuste de equações matemáticas para determinar a área foliar de mudas de Allagoptera arenaria de forma não destrutiva. International Journal of Development Research, v. 10, n. 3, p. 34399-34402, 2020.

PEDRO JÚNIOR, M. J.; RIBEIRO, I. J.; MARTINS, F. P. Determinação da área foliar em videira cultivar 'Niágara Rosada'. Bragantia, v.45, n.1, p.199-204, 1986.

POMPELLI, M. F.; ANTUNES, W. C.; FERREIRA D. T. R. G.; CAVALCANTE, P. G. S.; WANDERLEY FILHO, H. C. L.; ENDRES, L. Allometric models for nondestructive leaf area estimation of Jatropha curcas. Biomass and Bioenergy, v.36, p.77-85, 2012.

RAMKHELAWAN, E. \& BRATHWAITE, R. A. I. Leafarea estimation by nondestructive methods in sourorange (Citrus aurantium L.). Tropical Agriculture, v.67, n.3, p.203-206, 1990.

R CORE TEAM. R: a language and environment for statistical computing. Vienna: $\mathrm{R}$ Foundation for Statistical Computing, Vienna, Austria, 2020.

SANTOS, J. S. H.; SANTOS, K. T. H.; OLIVEIRA, V. S.; SANTOS, G. P.; MENEZES, L. F. T.; CZEPAK, M. P.; FALQUETO, A. R.; AOYAMA, E. M.; SCHMILDT, O.; SCHMILDT, E. R. Regression models for prediction of leaf area in purple ipe [Tabebuia impetiginosa (Mart.)]. Australian Journal of Crop Science, v. 12, n. 4, p. 654-659, 2020.

SCHMILDT, E. R.; AMARAL, J. A. T.; SANTOS, J. S.; SCHMILDT, O. Allometric model for estimating leaf area in clonal varieties of coffee (Coffea canephora). Revista Ciência Agronômica, v. 46, n. 4, p.740-748, 2015.

TEOBALDELLI, M.; BASILE, B.; GIUFFRIDA, F.; ROMANO, D.;
TOSCANO, S.; LEONARDI, C.; RIVERA, C. M.; COLLA, G.; ROUPHAEL. Y. Analysis of Cultivar-Specific Variability in Size-Related Leaf Traits and Modeling of Single Leaf Area in Three Medicinal and Aromatic Plants: Ocimum basilicum L., Mentha Spp., and Salvia Spp. Plants, v. 9, n.13, p.1-21, 2020.

WILLMOTT, C. J. On the validation of models. Physical Geography, v. 2, n.2, p. 184-194, 1981. https://doi.org/10.1080/02723646.1981.106 42213 